\title{
Mechanical Reliability of Reactively Alloyed NiAl as a Structural Material
}

\author{
Takahiro Namazu, ${ }^{*}$ Koichi Kuwahara, Masayuki Fujii, \\ Shunsuke Kanetsuki, ${ }^{1}$ Shugo Miyake, ${ }^{1}$ and Shozo Inoue \\ Department of Mechanical Engineering, University of Hyogo, 2167 Shosha, Himeji, Hyogo 671-2201, Japan \\ ${ }^{1}$ Kobelco Research Institute Inc., 1-5-5 Takatsukadai, Nishi-ku, Kobe, Hyogo 651-2271, Japan
}

(Received July 21, 2015; accepted August 21, 2015)

Keywords: Al/Ni multilayer film, $\mathrm{NiAl}$ alloy, exothermic reaction, reactive bonding, tensile test, bending test, mechanical reliability

In this study, for the practical use of reactively alloyed NiAl as a structural material, its mechanical reliability is evaluated. An Al/Ni multilayer film is attractive as a local heat source for soldering. To investigate the difference in mechanical characteristics between as-deposited $\mathrm{Al} /$ Ni multilayer films and reactively alloyed NiAl films, quasistatic uniaxial tensile tests and stress relaxation tests were conducted. A higher Young's modulus and a higher strength were obtained in the NiAl films. The films also showed better consistency under a constant applied strain. Then, four-point bending tests were carried out to examine the fracture strength of reactively bonded solder joints and to specify the fracture origin. All the joints fractured at the interface of the SnAg solder layer and the reactively alloyed NiAl layer. The durability of the reactively alloyed NiAl in the joints is discussed in the light of the grain size evaluated by electron backscatter diffraction (EBSD) analysis.

\section{Introduction}

Sputtered multilayer films consisting of the combination of a light metal and a transition metal, such as $\mathrm{Al} / \mathrm{Ni}, \mathrm{Al} / \mathrm{Ti}$, and $\mathrm{Ti} / \mathrm{Si}$, are known to show self-propagating exothermic reaction. ${ }^{(1-4)}$ By applying a small amount of energy, such as a spark, an electric power, and a pulse laser, they can start forming an intermetallic compound (IMC) at the interface of two different metal layers. Depending on the combination of materials, the reaction can happen even with a mechanical shock and a scratch. If an IMC is more stable than two independent materials in a multilayer film, the heat is definitely generated during the IMC formation. The generated heat at a given point is used for the next IMC formation in the surrounding portion; therefore, the exothermic phenomenon automatically propagates along the film. The advantages of the reaction are as follows: easy film preparation, easy ignition, flash heating, very fast exothermic reaction propagation, self-propagation of the reaction, controllable exothermic power, non-oxidation-reduction reaction, zero emissions, and low cost. ${ }^{(5-7)}$ On the basis of these advantages, we use sputtered $\mathrm{Al} / \mathrm{Ni}$ films as a localized heat source for the environment-friendly and low-cost manufacturing of solder joints. ${ }^{(8-11)}$ Since the films can generate the heat of reaction, approximately $1300 \mathrm{~J} / \mathrm{g}$ at maximum in every atmosphere, solder joints can be manufactured within a second in vacuum or inactive gas as well as ambient air.

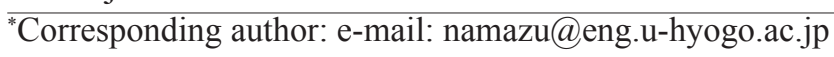


The mechanical properties and long-term durability of reactively bonded solder joints have to be investigated experimentally for obtaining a reliable design and lifetime estimation. To demonstrate the effectiveness of the reactive bonding technique, investigating the mechanical reliability of reactively alloyed NiAl IMC is significant because the IMC after bonding is sure to remain in the jointed portion. ${ }^{(1,2,7-11)}$ In this study, first, we performed quasi-static uniaxial tensile tests to compare the Young's modulus and fracture strength of reactively alloyed NiAl IMC specimens with those of as-deposited $\mathrm{Al} / \mathrm{Ni}$ multilayer film specimens. Stress relaxation tests were also carried out to understand the long-term durability of the two specimens. Then, four-point bending tests of single-crystal silicon (SCS) specimens containing a reactively bonded solder joint were conducted to examine the durability of the reactively alloyed NiAl IMC in the joint against external forces. The reliability of the IMC is discussed from the viewpoint of grain size using electron backscatter diffraction (EBSD) data.

\section{Experimental Procedure}

Figure 1 shows the process flow for fabricating a uniaxial tensile test specimen. We prepared two types of specimen, Al/Ni multilayer film and reactively alloyed NiAl film. These specimens are prepared by combining SCS frame fabrication and $\mathrm{Al} / \mathrm{Ni}$ multilayer film fabrication. First, a $300-\mu$ m-thick SCS (001) wafer is oxidized by wet thermal oxidation to grow a 1- $\mu$ m-thick $\mathrm{SiO}_{x}$ layer on the entire surface. After photolithography and wet etching to form a SCS frame, deep reactive ion etching (DRIE) is conducted. The SCS frame is composed of four SCS springs, two

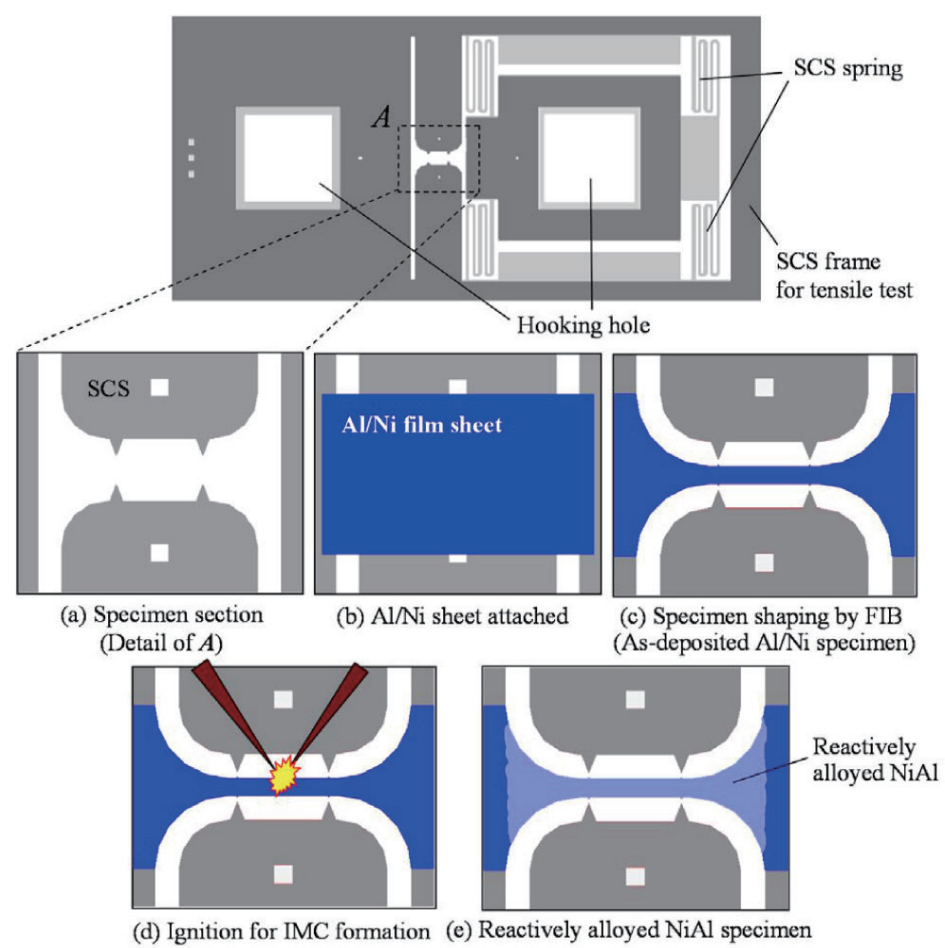

Fig. 1. (Color online) Schematic of fabrication process for as-deposited Al/Ni multilayer film specimen and reactively alloyed NiAl IMC specimen for uniaxial tensile tests and stress relaxation tests. 
hooking holes, and a through hole in the specimen section. ${ }^{(12-14)}$ Then, an Al/Ni multilayer film sheet is attached onto the specimen section with glue. An in-house ternary-source direct current (dc) magnetron sputtering equipment is used for the deposition of the Al/Ni multilayer film. The bilayer thickness and whole thickness are set to be $100 \mathrm{~nm}$ and $4 \mu \mathrm{m}$, respectively. The atomic ratio of Al to $\mathrm{Ni}$ is controlled to be $1: 1$, indicating that the thicknesses of $\mathrm{Al}$ and $\mathrm{Ni}$ are 60 and $40 \mathrm{~nm}$, respectively, because the enthalpy during the $\beta$-NiAl IMC formation is the largest among all the $\mathrm{NiAl}_{x}$ IMCs. The film is deposited onto a photoresist, used as a sacrificial layer, and then released by etching the photoresist with a remover. No damage to the $\mathrm{Al} / \mathrm{Ni}$ multilayer film is introduced by the etching. After the $\mathrm{Al} / \mathrm{Ni}$ sheet is attached, the sheet is fabricated to a "dog-bone" shape by focused ion beam (FIB) direct patterning. In this step, the fabrication of the as-deposited Al/Ni film specimen is completed. For the fabrication of the NiAl IMC specimen, a small spark induced by the contact of two bias-voltage-supplied probes is generated at the overhead of the Al/Ni specimen for ignition. Once the ignition is successfully made, the Al/Ni specimen reacts locally. Soon after that, the reaction rapidly propagates to the entire specimen; consequently, the reactively alloyed NiAl IMC specimen is completed.

Figure 2 shows photographs of the as-deposited $\mathrm{Al} / \mathrm{Ni}$ film specimen and the reactively alloyed NiAl film specimen produced for uniaxial tensile tests and stress relaxation tests. In the asdeposited $\mathrm{Al} / \mathrm{Ni}$ film specimen, a dog-bone shape is found to be formed precisely with FIB. On the entire area of the specimen, many small dark-colored dots can be seen. These are considered as redeposited materials that have fallen during the FIB fabrication. In the reactively alloyed NiAl specimen, it is found that the color and morphology of only the dog-bone section have changed from those of the $\mathrm{Al} / \mathrm{Ni}$ film specimen before the reaction. This indicates that only the changed portion has reacted by the application of a small spark. Note that the reacted portion is limited to the dog-bone section, indicating a self-standing $\mathrm{Al} / \mathrm{Ni}$ film portion in the specimen chip. By applying a spark, the reaction happened in the vicinity of the center of the self-standing portion, and then it propagated towards both ends of the specimen simultaneously. At both the grip ends, the propagation stopped owing to the heat released to the SCS substrate. ${ }^{(15)}$ The heat release gave rise to a reduction in thermal energy. The reduced thermal energy probably became less than the energy

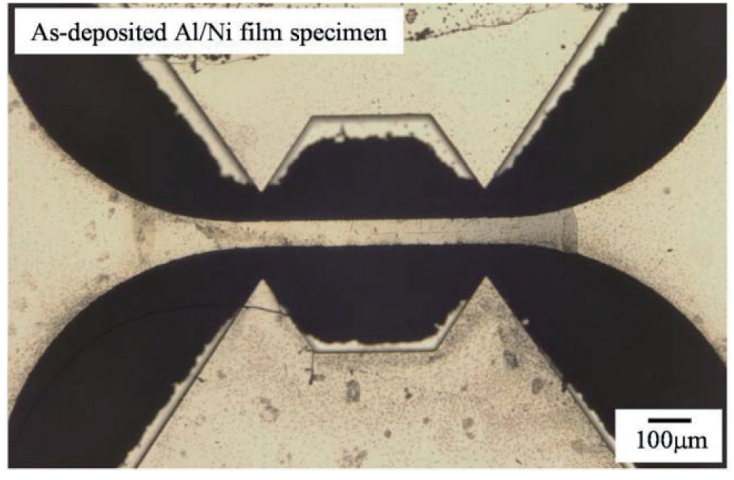

(a)

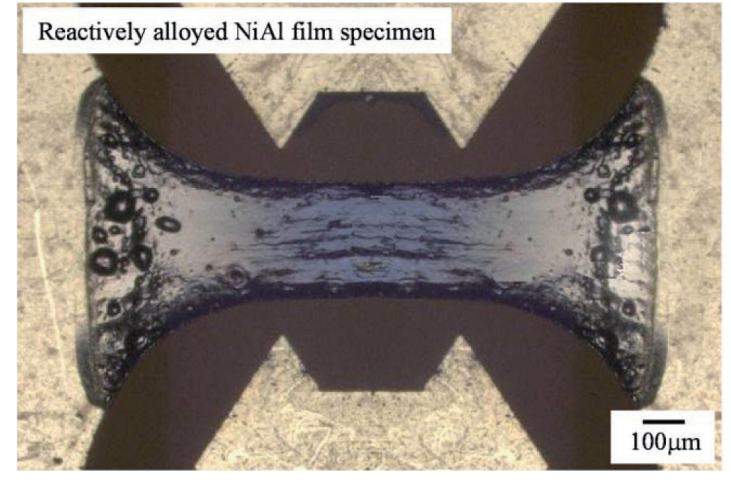

(b)

Fig. 2. (Color online) Photographs of (a) produced as-deposited Al/Ni multilayer film specimen and (b) reactively alloyed NiAl IMC specimen. From the photograph of the NiAl specimen, a self-propagating exothermic reaction for NiAl IMC formation is found to have happened in only a self-standing portion of the specimen chip. 
necessary for reaction propagation, so that only the self-standing portion reacted. All the reactively alloyed NiAl films consist of the ordered B2-type NiAl intermetallic compound in the $\beta$-NiAl phase because the atomic ratio of $\mathrm{Al}$ to $\mathrm{Ni}$ was 1:1. In the two types of specimen, the width and length were set to range from $50 \mu \mathrm{m}$ and $500 \mu \mathrm{m}$ to $950 \mu \mathrm{m}$ and $3 \mathrm{~mm}$, respectively. The thickness of all the specimens was almost $4 \mu \mathrm{m}$ with or without the reaction.

An in-house uniaxial tensile test equipment was used for quasi-static tensile tests and stress relaxation tests. The details of the equipment are published elsewhere. ${ }^{(13,16-19)}$ The equipment consists of a piezoelectric actuator, an actuator case with a lever for elongation amplification, a load cell with a resolution of $2 \mathrm{mN}$, a linear variable differential transformer (LVDT) for tension stroke measurement, an image analysis system with a charge-coupled device (CCD) camera for the specimen's displacement measurement, and a specimen holder with a microheater. The image analysis system has a resolution of $13 \mathrm{~nm} /$ pixel for the displacement measurement. All the quasistatic tensile tests were performed under a constant strain rate of $0.002 \mathrm{~s}^{-1}$ in ambient air.

Figure 3 shows a schematic of the process flow for fabricating a reactively bonded solder joint specimen along with a photograph of the four-point bending test. First, a 1.5-mm-thick SCS (001) wafer is mechanically cut by blade dicing to make two SCS chips with different sizes of $10 \times 10$ $\mathrm{mm}^{2}$ and $10 \times 12 \mathrm{~mm}^{2}$, which are used as the top and base chips for soldering, respectively. A different chip size enables us to easily access the $\mathrm{Al} / \mathrm{Ni}$ reactive layer with two electrode probes for ignition. A 500-nm-thick Ni plus 50-nm-thick Cr double interlayer is then deposited by dc magnetron sputtering onto both the SCS chips after rinsing with pure water. After the deposition, a 2- or $6-\mu \mathrm{m}$-thick $\mathrm{Sn}-3.5 \mathrm{Ag}$ solder film is deposited by dc magnetron cosputtering onto the interlayer of the two chips. After that, a 30- $\mu \mathrm{m}$-thick Al/Ni multilayer film with a bilayer thickness of $100 \mathrm{~nm}$ is deposited by alternative dc magnetron sputtering onto the base chip only. The atomic ratio of $\mathrm{Al}$ to $\mathrm{Ni}$ is set to be 1:1 as with all the tensile specimens. After these processes, the top chip is placed onto the base chip facing each other, followed by applying a pressure load of $200 \mathrm{kgf}$ ( 2 $\mathrm{kgf} / \mathrm{mm}^{2}$ ) to the overlapped chips. An electrical shock is supplied directly to the Al/Ni film with two probes made of cemented carbide, to activate it for melting solder layers in vacuum. ${ }^{(9-11)}$ The

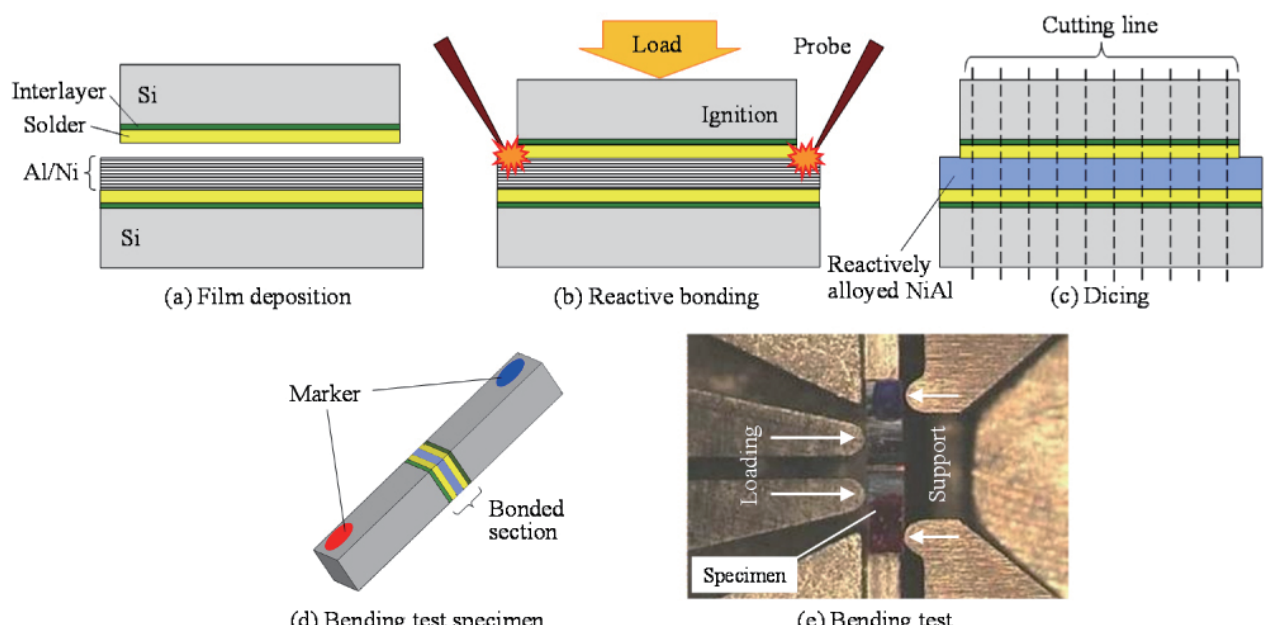

(d) Bending test specimen

(e) Bending test

Fig. 3. (Color online) Schematic of fabrication process for reactively bonded solder joint specimen for four-point bending tests. 
electric voltage and current for ignition are set to be $10 \mathrm{~V}$ and $0.1 \mathrm{~A}$, respectively. After the reactive bonding, the bonded chips are mechanically cut by blade dicing to produce rectangular solid specimens, measuring $0.5 \times 0.5 \times 3 \mathrm{~mm}^{3}$, having the bonded section at the midpoint. All the diced specimens are carefully polished by hand to remove unwanted cutter marks affecting failure before the strength tests. Finally, blue and red markers are painted on both ends of the specimens by hand to differentiate the top and base SCS chips, respectively.

The produced specimens are set into the sample holder in an in-house four-point bending test equipment, as shown in Fig. 3(e). This equipment (not shown here) is composed of a piezoelectric actuator in an actuator case, a load cell with a resolution of $10 \mathrm{mN}$, a laser displacement meter with a resolution of $1 \mathrm{~nm}$, an image analysis system with a CCD camera for the observation of specimen deformation, a sample holder with an $X-Y-Z$ stage, and loading and supporting styluses with a tip radius of $0.3 \mathrm{~mm} . .^{(10,20)}$ The loading and supporting styluses are made of cemented carbide to minimize as much as possible unwanted elastic deformation during the test. The distance between the two loading styluses is $1.0 \mathrm{~mm}$, and the distance between the two supporting styluses is $2.4 \mathrm{~mm}$. Since the reactively bonded SCS specimens are composed of multiple layers made of different types of material, we cannot predict where the specimens will fracture during the test. Thus, we have adopted the four-point bending test that makes it possible to apply a uniform bending moment to the specimens between the two loading styluses. Applying uniform bending stress to the bonded section allows us to know the weakest point between the styluses. The maximum bending stress $\sigma_{\max }$ produced at the top surface of the bonded section is given by ${ }^{(21)}$

$$
\sigma_{\max }=\frac{3 P\left(l_{2}-l_{1}\right)}{2 w t^{2}}
$$

where $P$ is the bending force, $l_{1}$ is the distance between the two loading styluses, $l_{2}$ is the distance between the two supporting styluses, $w$ is the specimen width, and $t$ is the specimen thickness. For all the specimens, the loading speed was kept constant at $10 \mu \mathrm{m} / \mathrm{min}$. All the tests were implemented in ambient air.

\section{Results and Discussion}

Figure 4 shows representative tensile stress-strain relations for the as-deposited $\mathrm{Al} / \mathrm{Ni}$ film specimen and reactively alloyed NiAl film specimen, which are represented as the black and gray solid lines, respectively. The stress-strain relation of the as-deposited $\mathrm{Al} / \mathrm{Ni}$ film specimen is almost linear, although at the beginning of the test, a slight nonlinear region is observed. When the strain is around $0.55 \%$, it is found that the specimen has fractured in a brittle manner. In the reactively alloyed NiAl film specimen, the stress-strain relation seems to be almost linear as well. A brittle fracture occurred as with the specimen before the reaction.

Table 1 lists the Young's modulus and tensile strength of the as-deposited $\mathrm{Al} / \mathrm{Ni}$ and reactively alloyed NiAl specimens. The numbers of the specimens tested before and after the reaction are 12 and 10, respectively. The mean Young's modulus of the as-deposited specimen is $117.0 \mathrm{GPa}$, which is independent of the width and length of the specimen tested here. The mean value is found to be located between the Young's moduli of Al and Ni. Assuming that the deformation of the multilayer specimen obeys the linear elastic theory for isotropic materials, the effective Young's modulus $E_{1+2}$ 


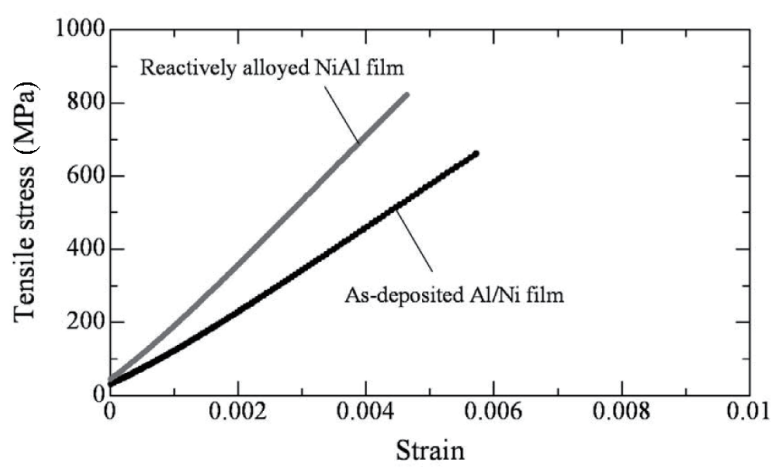

Fig. 4. Representative tensile stress-strain relations for as-deposited $\mathrm{Al} / \mathrm{Ni}$ multilayer film specimen and reactively alloyed NiAl IMC specimen obtained from quasi-static uniaxial tensile tests.

Table 1

Summary of Young's modulus and tensile strength obtained from quasi-static uniaxial tensile tests.

\begin{tabular}{lccccc}
\hline \multirow{2}{*}{ Specimen } & Number of & \multicolumn{2}{c}{ Young's modulus } & \multicolumn{2}{c}{ Tensile strength } \\
\cline { 3 - 5 } & specimens & $E(\mathrm{GPa})$ & Stdev $(\mathrm{GPa})$ & $\sigma_{\mathrm{f}}(\mathrm{MPa})$ & Stdev $(\mathrm{MPa})$ \\
\hline As-deposited Al/Ni film & 12 & 117.0 & 8.4 & 615.8 & 32.3 \\
Reactively alloyed NiAl film & 10 & 171.2 & 9.9 & 895.6 & 46.6 \\
\hline
\end{tabular}

can be expressed as ${ }^{(22)}$

$$
E_{1+2}=\frac{t_{1}}{t_{1}+t_{2}} \cdot \frac{E_{1}}{1-v_{1}^{2}}+\frac{t_{2}}{t_{1}+t_{2}} \cdot \frac{E_{2}}{1-v_{2}^{2}}-\frac{\left(\frac{t_{1}}{t_{1}+t_{2}} \cdot \frac{E_{1} v_{1}}{1-v_{1}^{2}}+\frac{t_{2}}{t_{1}+t_{2}} \cdot \frac{E_{2} v_{2}}{1-v_{2}^{2}}\right)^{2}}{\frac{t_{1}}{t_{1}+t_{2}} \cdot \frac{E_{1}}{1-v_{1}^{2}}+\frac{t_{2}}{t_{1}+t_{2}} \cdot \frac{E_{2}}{1-v_{2}^{2}}}
$$

where $E$ and $v$ are the Young's modulus and Poisson's ratio, respectively. The subscripts 1 and 2 are indicative of $\mathrm{Al}$ and $\mathrm{Ni}$ or vice versa, respectively. $t$ is the total thickness for each material in a film. In the case of the as-deposited $\mathrm{Al} / \mathrm{Ni}$ film specimen used in this study, the thicknesses of $\mathrm{Al}$ and $\mathrm{Ni}$ in a bilayer are 60 and $40 \mathrm{~nm}$, respectively, because the atomic ratio has been set to be 1:1. Thus, the total thicknesses of $\mathrm{Al}$ and $\mathrm{Ni}$ in the film are calculated to be 2.4 and $1.6 \mu \mathrm{m}$, respectively. According to previous reports, the nominal Young's modulus and Poisson's ratio for $\mathrm{Al}$ are 72 $\mathrm{GPa}$ and 0.34 , respectively. ${ }^{(23,24)} \mathrm{For} \mathrm{Ni}$, those values are $191 \mathrm{GPa}$ and 0.30 , respectively. ${ }^{(25)}$ By substituting those literature values and the thicknesses into Eq. (2), the effective Young's modulus can be estimated as $119.6 \mathrm{GPa}$. The experimentally obtained value deviates by only $-2.2 \%$ from the estimated value, which indicates a good uniformity of the Al/Ni multilayer film, a good workability of the specimen with FIB, and a good precision of the force and displacement measurements during the tests. For the reactively alloyed NiAl specimen, the mean Young's modulus is $171.2 \mathrm{GPa}$, which is approximately 1.46 times higher than that of the as-deposited film specimen. The mean value is almost comparable to the literature value for NiAl bulk. ${ }^{26-29)}$ The tensile strength of the $\mathrm{NiAl}$ specimen is $895.6 \mathrm{MPa}$ on average, which is around $45 \%$ higher than that of the as-deposited $\mathrm{Al} / \mathrm{Ni}$ specimen. From these results, it is found that the reactive alloying of the Al/Ni multilayer film is able to simply produce a NiAl IMC film having excellent mechanical properties. 
Figure 5 shows representative scanning electron microscopy (SEM) images of the as-deposited and reactively alloyed film specimens. The fracture surfaces of both specimens are found to be perpendicular to the longitudinal direction. In the as-deposited film specimen, the multiple layers made of $\mathrm{Al}$ and $\mathrm{Ni}$ are clearly seen. The thickness of each layer appears very uniform throughout the film, which is evidence that the multilayer film has been fabricated finely by alternative dc magnetron sputtering by controlling the electric power and deposition time. A dimplelike pattern can be detected on the surface, which implies that plastic deformation might have happened very slightly just before the fracture, although no nonlinear region has been found in the stress-strain relation. In the reactively alloyed film specimen, the film structure is found to contain crystal grains, indicating that the $\mathrm{Al} / \mathrm{Ni}$ changed; that is, the surface after the reaction appears more irregular than that before the reaction. The crystal grains are considered to be probably NiAl IMC, according to the atomic ratio of the as-deposited $\mathrm{Al} / \mathrm{Ni}$ film. The grain size is within the range from $300 \mathrm{~nm}$ to $1 \mu \mathrm{m}$, which is roughly comparable to the wavelength of the concavo-convex pattern on the surface. Therefore, the surface irregularity would have been brought about by the grain growth, and owing to intercrystalline cracking derived from the stress concentration around the concave portion, the reactively alloyed NiAl film would have fractured in a brittle manner.

Figure 6 shows representative results of stress relaxation tests. The black and gray solid lines are indicative of the relaxation curves for the as-deposited and reactively alloyed film specimens, respectively. To make a tensile strain constant throughout each test, the elongation measured with LVDT was kept constant using a feedback system. The initial tensile stresses were set to be 400 and $160 \mathrm{MPa}$ for the two specimens. When the initial stress is $400 \mathrm{MPa}$, the stress rapidly drops at the start of the test for the as-deposited specimen. At $500 \mathrm{~s}$, the stress is found to be around 300 $\mathrm{MPa}$, indicating a $100 \mathrm{MPa}$ drop from the initial value. After that, the stress gradually decreases with time. At $3000 \mathrm{~s}$, the stress reaches $260 \mathrm{MPa}$, which is a $35 \%$ reduction from the initial stress. For the reactively alloyed specimen, the stress reduction rate is relatively low compared with that
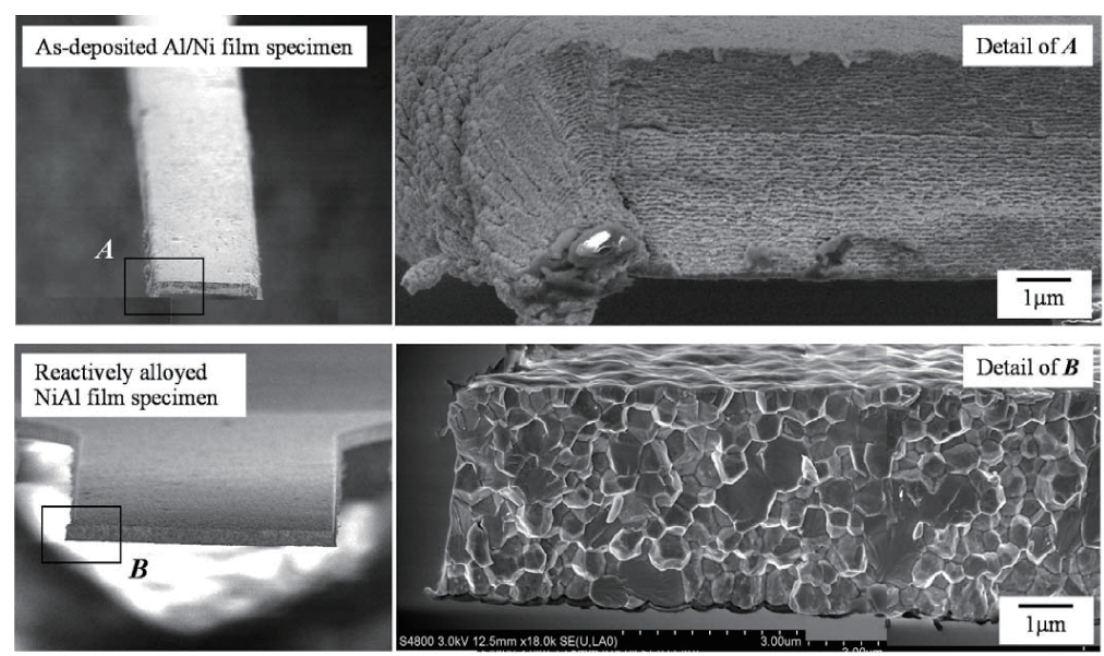

Fig. 5. SEM images of fracture surface for as-deposited Al/Ni multilayer film specimen and reactively alloyed $\mathrm{NiAl}$ IMC specimen. By exothermic reaction, it is found that the Al/Ni multilayer structure disappeared, and alternatively, NiAl crystal grains appeared in the reactively alloyed NiAl IMC specimen. 


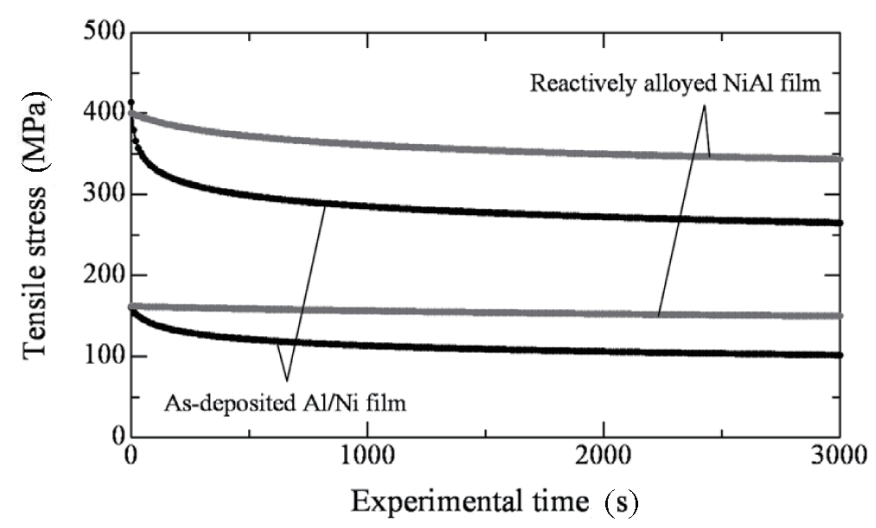

Fig. 6. Representative stress relaxation curves for as-deposited $\mathrm{Al} / \mathrm{Ni}$ multilayer film specimen and reactively alloyed NiAl IMC specimen.

for the as-deposited specimen. Even at $3000 \mathrm{~s}$, the stress is $345 \mathrm{MPa}$, which is only a $14 \%$ drop from the initial value. The amount of stress reduction is approximately $39 \%$ of that for the asdeposited specimen. When the initial stress is $160 \mathrm{MPa}$, almost the same trend as that for $400 \mathrm{MPa}$ can be seen. Note that for the reactively alloyed specimen, the stress hardly drops even at $3000 \mathrm{~s}$. The amount of stress reduction is merely $5 \mathrm{MPa}$, which is only a $3.1 \%$ drop from the start point, and is one-eleventh of that for the same specimen at the initial stress of $400 \mathrm{MPa}$. Compared with the as-deposited specimen, the amount of stress reduction for the reactively alloyed specimen is only one-twelfth. Therefore, it has been confirmed that the reactively alloyed NiAl IMC film is more durable against constantly applied external forces than the as-deposited Al/Ni film.

From the results of quasi-static tensile tests and stress relaxation tests, it was found that the reacted NiAl IMC film has superior mechanical characteristics and superior long-term durability compared with the $\mathrm{Al} / \mathrm{Ni}$ multilayer film before the reaction. To investigate the mechanical reliability of the reacted NiAl IMC layer in reactively bonded solder joints, four-point bending tests of the jointed specimens were conducted [see Figs. 3(d) and 3(e)]. Figure 7 shows representative bending stress-deflection relations of the specimens with 2- and 12- $\mu$ m-thick SnAg solder layers, which are plotted using the dark and light gray points, respectively. The stress-deflection data for the two specimens are found to show large scatter compared with the stress-strain data shown in Fig. 4. This is because the deflection measured with a laser displacement meter has been scattered. On the specimen surface, an irregular reflection originating from blade-dicing-derived cutter marks or incomplete polishing would have yielded the data spread. The stress-deflection relation for the specimen including the $2-\mu \mathrm{m}$-thick solder layer is found to be linear despite the data scattering. When the stress of $28 \mathrm{MPa}$ was produced at the center of the specimen, the specimen fractured in a brittle matter. In the case of the solder thickness of $12 \mu \mathrm{m}$, the slope of the relation is smaller than that in the thinner solder specimen. This would be caused by the difference in the amount of deformation between the 2- and $12-\mu \mathrm{m}$-thick solder layers. The bending strength of the $12-\mu \mathrm{m}-$ thick solder specimen is $18 \mathrm{MPa}$, about $36 \%$ lower than that of the $2-\mu \mathrm{m}$-thick solder specimen.

The bending test results are summarized in Table 2. The number of specimens tested is 10 for each type of specimen. The mean bending strength of the $2-\mu \mathrm{m}$-thick solder specimen is $32.3 \mathrm{MPa}$, 


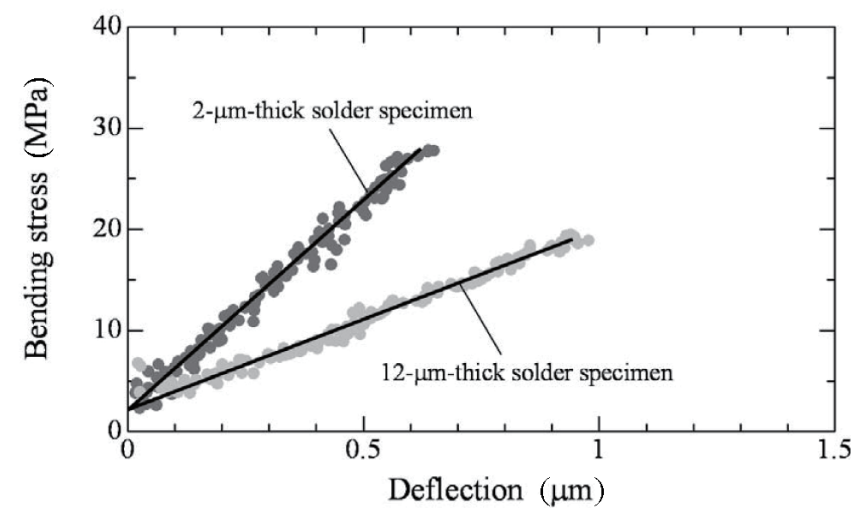

Fig. 7. Representative bending stress-deflection relations for as-deposited $\mathrm{Al} / \mathrm{Ni}$ multilayer film specimen and reactively alloyed NiAl IMC specimen derived from four-point bending tests.

Table 2

Summary of bending strength obtained from four-point bending tests.

\begin{tabular}{ccccc}
\hline Solder thickness & Number of & Interlayer & \multicolumn{2}{c}{ Bending strength } \\
\cline { 4 - 5 }$t(\mu \mathrm{m})$ & specimens & Cr/Ni $(\mathrm{nm})$ & $\sigma_{\mathrm{b}}(\mathrm{MPa})$ & Stdev $(\mathrm{MPa})$ \\
\hline 2 & 10 & \multirow{2}{*}{$50 / 500$} & 32.3 & 2.8 \\
12 & 10 & & 20.3 & 2.9 \\
\hline
\end{tabular}

which is approximately 1.6 times higher than that of the $12-\mu \mathrm{m}$-thick solder specimen. This solder thickness effect means that the solder layer has possibly affected the specimen's fracture as well as deformation. On the other hand, the standard deviation for each specimen shows almost the same value, implying that there might be no difference in the fracture mechanism between the two specimens. In the side-view photographs at the bonded section of these specimens, as presented in Fig. 8, no obvious cracks and voids are seen in the 2- $\mu$ m-thick solder specimen, whereas it is found that many cracks and voids exist in the reactively alloyed NiAl layer of the $12-\mu \mathrm{m}$-thick solder specimen. According to energy-dispersive X-ray (EDX) images of the top side fracture surface in the 2- $\mu \mathrm{m}$-thick solder specimen, a large crack growth from the lower left corner to the inside is seen. The specimen would have fractured at the corner where the maximum tensile stress was produced during bending. In the fracture surface images of the 12- $\mu \mathrm{m}$-thick solder specimen, no cracks are found, but in the base side, the surface is found to be rougher than that for the 2- $\mu \mathrm{m}$-thick solder specimen. This would be attributed to the easiness of the reactive layer's deformation in the out-of-plane direction during the IMC formation involving volume shrinkage. Thicker solder films sandwiching an $\mathrm{Al} / \mathrm{Ni}$ reactive layer would be able to tolerate the $\mathrm{Al} / \mathrm{Ni}$ 's volume shrinkage more than thinner solder films. ${ }^{(30)}$ Note that the fracture surfaces in both specimens are mainly composed of $\mathrm{Ni}$ and $\mathrm{Sn}$. That is, the two specimens have fractured at the interface of the reactively alloyed NiAl layer and the SnAg solder layer with or without the defects in the NiAl IMC layer. This experimental fact strongly demonstrates that the reactively alloyed NiAl IMC layer in a reactively bonded solder joint is definitely stronger than the NiAl-SnAg interface as well as the SnAg solder layer. In fact, as listed in Table 1, the fracture strength of the reactively alloyed NiAl films obtained 

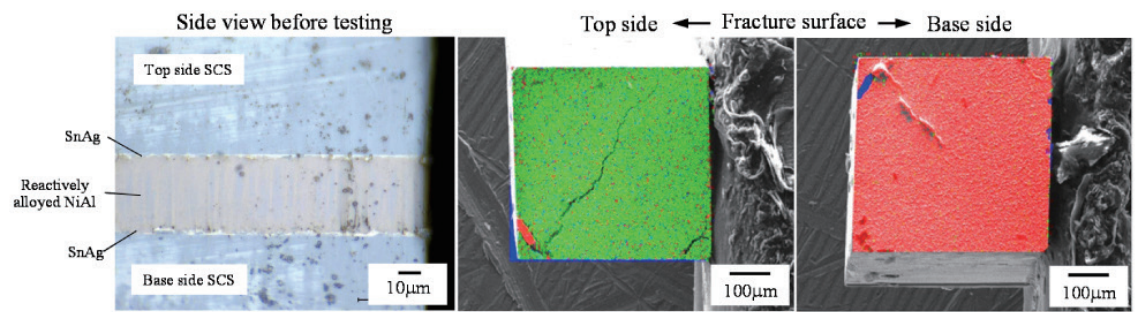

2 - $\mu \mathrm{m}$-thick solder specimen

$\square \mathrm{Sn} \square \mathrm{Ni} \square \mathrm{Si}$

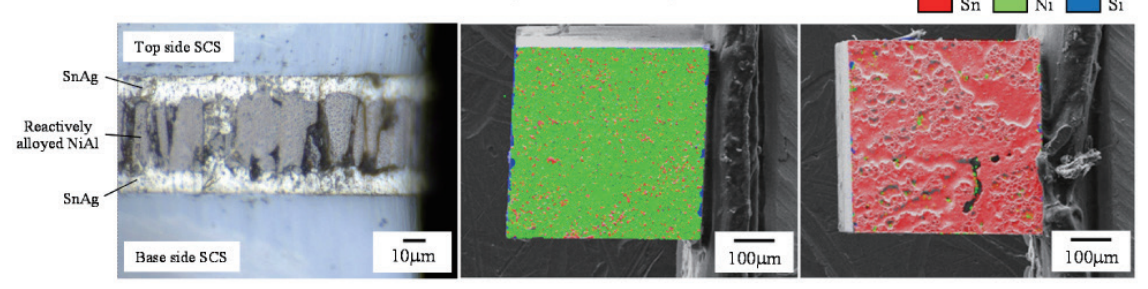

12- $\mu$ m-thick solder specimen

Fig. 8. (Color online) Side-view photographs of jointed section in the specimens with 2- and 12- $\mu$ m-thick $\mathrm{SnAg}$ solder layers along with EDX maps of fracture surfaces.

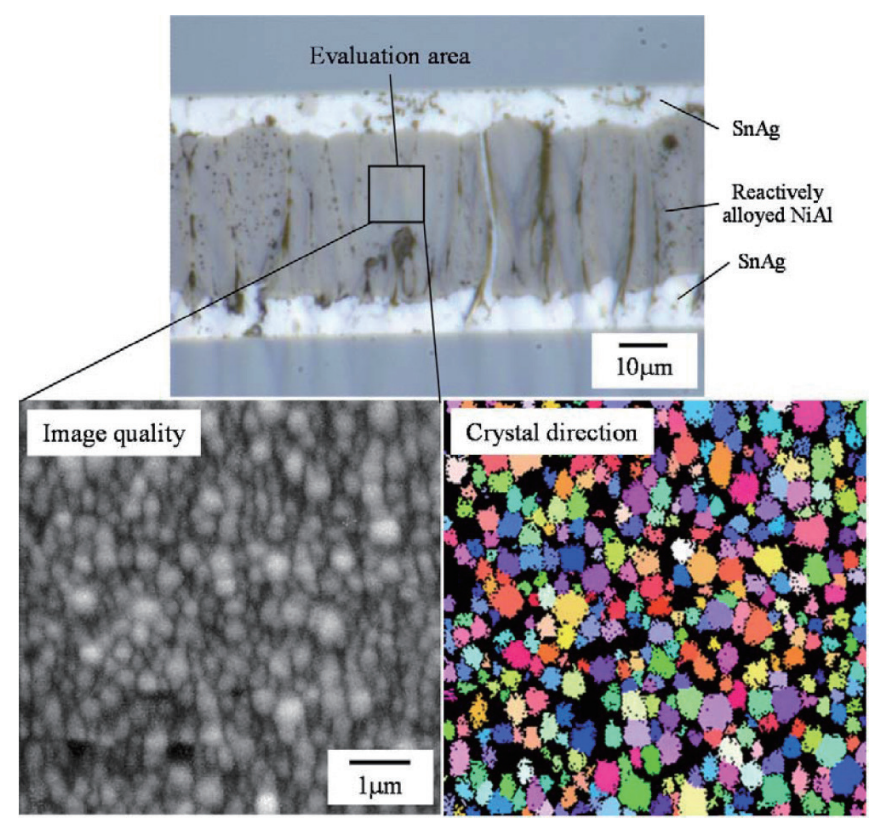

Fig. 9. (Color online) Image quality map and crystal direction map in reactively alloyed NiAl IMC of the jointed specimen derived from EBSD analysis.

from quasi-static tensile tests is $895.6 \mathrm{MPa}$ on average, which is amazingly $27.7-44.1$ times higher than that of the reactively bonded solder joints, including the reactively alloyed NiAl IMC layer. Moreover, from the image quality map derived from EBSD analysis shown in Fig. 9, the reactively alloyed NiAl layer of the joint is found to be composed of submicron-size crystal grains. This can be confirmed from the crystal direction map showing that the crystal directions are randomly oriented. The grain size is found to range from sub-100 to $300 \mathrm{~nm}$, roughly one-third of the grain 
size in the self-standing reactively alloyed NiAl film used for the tensile tests (see Fig. 5). The difference in grain size is related to the amount of heat released during the reaction. In the case of reactive solder joint fabrication, the generated heat during the exothermic reaction was probably conducted rapidly to the SCS chips through the SnAg solder layer because SCS and SnAg have relatively high heat conductivities. Consequently, the reactively alloyed NiAl layer would have quickly cooled, and the cooling rate would have been definitely faster than that in a self-standing $\mathrm{NiAl}$ tensile specimen. The fast cooling would have led to the prevention of crystal grain growth in the NiAl layer. Typically, a metallic alloy consisting of small grains is well known to be stronger than that consisting of large grains. ${ }^{(31,32)}$ From this, the intrinsic strength of the reactively alloyed NiAl IMC in the solder joint is possibly higher than that of the self-standing reactively alloyed $\mathrm{NiAl}$ film. Therefore, the reactively alloyed NiAl layer in the joints can be considered to be reliable mechanically, and the reactive bonding process for solder joints would be effective for practical use if the adhesion between the reactively alloyed NiAl and the SnAg solder is further strengthened.

\section{Conclusion}

We investigated the mechanical reliability of reactively alloyed NiAl IMC evaluated by quasistatic tensile tests, stress relaxation tests, and four-point bending tests. Al/Ni multilayer films and reactively alloyed NiAl films showed brittle failure without plastic deformation. The Young's modulus and fracture strength of the NiAl film specimens after exothermic reaction were higher than those of the Al/Ni film specimens before the reaction. Stress relaxation tests demonstrated that the reactively alloyed NiAl specimens possessed a long-term mechanical durability compared with the Al/Ni multilayer film specimens. The strength of the reactively bonded solder joint specimens was evaluated by four-point bending tests. All the joint specimens fractured at the interface of the reactively alloyed NiAl layer and the $\mathrm{SnAg}$ solder layer, and no fractures at the NiAl layer were found. The EBSD analysis suggested that the grain size was smaller by roughly one-third in the NiAl layer than in the free-standing reactively alloyed NiAl film. The reactively alloyed NiAl IMC was found to have superior mechanical characteristics even when the IMC remained in solder joints; therefore, the reactive bonding process will be utilizable as a future reliable fabrication technique for solder joints.

\section{References}

1 J. Wang, E. Besnoin, A. Duckham, S. J. Spey, M. E. Reiss, O. M. Knio, M. Powers, M. Whitener, and T. P. Weihs: Appl. Phys. Lett. 83 (2003) 3987.

2 J. Wang, E. Besnoin, A. Duckham, S. J. Spey, M. E. Reiss, O. M. Knio, and T. P. Weihs: J. Appl. Phys. 95 (2004) 248.

3 G. M. Fritz, S. J. Spey, Jr., M. D. Grapes, and T. P. Weihs: J. Appl. Phys. 113 (2013) 014901.

4 S. Minamibata, T. Namazu, K. Yoshiki, and S. Inoue: Proceedings of the 12th Int. Symp. on Sputtering and Plasma Processes (2013) pp. 299-302.

5 J. C. Trenkle, L. J. Koerner, M. W. Tate, S. M. Gruner, T. P. Weihs, and T. C. Hufnagel: Appl. Phys. Lett. 93 (2008) 081903.

6 R. Knepper, M. R. Snyder, G. Fritz, K. Fisher, O. M. Knio, and T. P. Weihs: J. Appl. Phys. 105 (2009) 083504.

7 B. Boettge, J. Braeuer, M. Wiemer, M. Petzold, J. Bagdahn, and T. Gessner: J. Micromech.Microeng. 20 (2010) 064018.

8 T. Namazu, H. Takemoto, H. Fujita, Y. Nagai, and S. Inoue: Proceedings of the 19th IEEE Int. Conf. on Microelectromech. Syst. (2006) pp. 286-289. 
9 H. Fujita, T. Namazu, and S. Inoue: Extended Abstracts of the 7th Int. Conf. of Advanced Technology in Experimental Mechanics (2007) OS05-3-1.

10 T. Namazu, K. Ohtani, S. Inoue, and S. Miyake: J. Eng. Mater. Technol. 137 (2015) 031011.

11 S. Miyake, S. Kanetsuki, K. Morino, J. Kuroishi, and T. Namazu: Jpn. J. Appl. Phys. 54 (2015) 06FP15.

12 T. Namazu, Y. Nagai, N. Naka, N. Araki, and S. Inoue: J. Eng. Mater. Technol. 134 (2011) 011009.

13 M. Fujii, T. Namazu, H. Fujii, K. Masunishi, Y. Tomizawa, and S. Inoue: J. Vac. Sci. Technol. B 30 (2012) 031804.

14 T. Namazu, M. Fujii, H. Fujii, K. Masunishi, Y. Tomizawa, and S. Inoue: J. Microelectromech. Syst. 22 (2013) 1414.

15 S. Ito, S. Inoue, and T. Namazu: Proceedings of the 17th Int. Conf. on Solid-State Sensors, Actuators and Microsystems, 2013, pp. 1927-1930.

16 T. Namazu, S. Inoue, H. Takemoto, and K. Koterazawa: IEEJ Trans. Sens. Micromachines 125 (2005) 374.

17 T. Namazu and Y. Isono: J. Microelectromech. Syst. 18 (2009) 129.

18 M. Komatsubara, T. Namazu, Y. Nagai, S. Inoue, N. Naka, S. Kashiwagi, and K. Ohtsuki: Jpn. J. Appl. Phys. 48 (2009) $04 \mathrm{C} 021$.

19 T. Namazu, H. Takemoto, and S. Inoue: Sens. Mater. 22 (2010) 13.

20 T. Namazu, T. Ishikawa, and Y. Hasegawa: J. Mater. Sci. 46 (2011) 3046.

21 M. Huurman and A. C. Pronk: Advanced Testing and Characterization of Bituminous Materials, eds. A. Loizos, M. N. Partl, T. Scarpas and I. L. Al-Qadi (CRC Press, Boca Raton, 2009) Vol. 2, p. 749.

22 K. K. Chawla: Composite Materials, Science and Engineering (Springer-Verlag, New York, 1998) p. 207.

23 Y. Kabe, H. Tanimoto, and H. Mizubayashi: Mater. Trans. 45 (2004) 119.

24 E. Eiper, R. Resel, C. Eisenmanger-Sittner, M. Hafok, and J. Keckes: Advances in X-ray Analysis 47 (2004) 368.

25 D. C. Hurley, R. H. Geiss, M. Kopycinska-Müller, J. Müller, D. T. Read, J. E. Wright, N. M. Jennett, and A.S. Maxwell: J. Mater. Res. 20 (2005) 1186.

26 D. B. Miracle and R. Darolia: Intermetallic Compounds, eds J. H. Westbrook and R. L. Fleischer (Wiley, NJ, 1995) Vol. 2, Chap. 3, p. 55.

27 G. Frommeyer and R. Rablbauer: Steel Research International 79 (2008) 507.

28 J. Lu, Q. Hu, and R. Yang: J. Mater. Sci. Technol. 25 (2009) 215.

29 M. Chmielewski, S. Nosewicz, K. Pietrzak, J. Rojek, A. Strojny-Nedza, S. Mackiewicz, and J. Dutkiewicz: J. Mater. Eng. Perform. 23 (2014) 3875.

30 T. Namazu, K. Ohtani, K. Yoshiki, and S. Inoue: Proceedings of the 16th Int. Conf. on Solid-State Sensors, Actuators and Microsystems (2011) pp. 1368-1371.

31 I. Baker, P. Nagpal, F. Liu, and P. R. Munroe: Acta Metallurgica et Materialia 39 (1991) 1637.

32 Y. N. Wang and J. C. Huang: Mater. Trans. 48 (2007) 184. 